\title{
Triggering Network Services Through Context-Tagged Flows
}

\author{
Roel Ocampo ${ }^{1,2}$, Alex Galis ${ }^{2}$, and Chris Todd ${ }^{2}$ \\ 1 Department of Electrical and Electronics Engineering, University of the Philippines, \\ Diliman, Quezon City, 1101 Philippines \\ 2 Department of Electronic and Electrical Engineering, University College London, \\ Torrington Place, London WC1E 7JE \\ $\{$ r.ocampo, a.galis, c.todd $\}$ @ee.ucl.ac.uk
}

\begin{abstract}
Next-generation Grids will require flexible and adaptive network infrastructures that would be able to provide the requisite quality of service for computational flows. We discuss a mechanism where network flows are tagged with network context information, triggering dynamic network services, including QoS and adaptation services. An incremental deployment strategy and some initial results are presented.
\end{abstract}

\section{Introduction}

Due to the nature of the computational tasks that grids handle, the underlying resources they use are required to deliver nontrivial qualities of service [1]. In networked environments, where resources such as bandwidth are often shared, there may be situations where computational flows would have to compete for the use of resources along with flows from less critical applications. To deal with this, a pragmatic approach might be to differentiate the various network flows and set an adaptation policy $P$ on a particular group of flows based on the class of users $U$ generating the flow, for a particular type of usage or activity $A$, under a certain situation or set of network conditions $C$. In other words, an adaptation $P$ is triggered by the general parameters $(U, A, C)$.

Currently the mechanisms that exist to differentiate network flows provide very limited information about the flow, and are usually non-extensible. In this work, we propose the use of rich and extensible information in the form of network context as a means of differentiating flows and triggering network services, particularly adaptation services. In Sect. 2 we argue that end-hosts are rich sources of information needed in triggering adaptation services, and that a mechanism for sharing such information with the rest of the network is needed. In Sect. 3 we present the concept and design for such a mechanism, called context tagging, and Sect. 4 briefly discusses an incremental deployment strategy. Section 5 discusses some initial results. Section 6 briefly discusses efforts that are related to our current work, and finally Sect. 7 concludes and describes the work ahead. 


\section{End-Hosts and Flow Context}

As a consequence of the end-to-end principle [2], nodes such as routers often have a very limited view of the state of the network. While they often have a global view of the network's topological state as a result of routing exchanges, routers may have a limited, per-node awareness of other parameters such as traffic levels, congestion, or bit error rates. Other information that may characterize the state of the network, such as path loss, delay and jitter are usually sensed and processed at end-hosts.

Aside from end-to-end traffic-related parameters, end-hosts are rich sources of other types of information such as the characteristics and capabilities of enddevices, the applications generating the traffic, and the identities and activities of users such as their movement and location. All of these pieces of information are relevant in (a) determining whether a certain condition $C$ exists within the network or user environment, (b) in identifying whether the traffic belongs to the class of users $U$, and (c) if the traffic is the result of usage activity $A$. These parameters also help in the design of appropriate adaptation policies applicable to the user group under these circumstances, or within that particular context.

\subsection{The Context of a Flow}

Context is defined in the New Oxford Dictionary of English as "the circumstances that form the setting for an event ... and in terms of which it can be fully understood and assessed." Dey, Salber and Abowd further define context as "any information that can be used to characterize the situation of entities ... that are considered relevant to the interaction between a user and an application, including the user and the applications themselves" [3]. Although the latter definition is tailored to interactions between users and applications, it can still be used as a template to describe the notion of context relevant to the interaction between a user application and the network, or between a user and the network.

One entity we consider relevant to this interaction is the flow, defined as distinguishable streams of related datagrams, typically resulting from the activity of a single entity [4]. The fact that flows are attributable to specific user groups and their activities makes them ideal sources of adaptation triggers $U$ and $A$. We define the context $C_{f}$ of a network flow as any information that can be used to characterize its situation, including information pertaining to other entities and circumstances that give rise to or accompany its generation at the source, affect its transmission through the network, and influence its use at its destination. This collectively includes not only the intrinsic, "low-level" characteristics of a flow, such as its traffic profile, but also the nature of the applications, devices, and the activities and identities of the users that produce or consume the flow.

We envision flow context to be used within the network in the following ways:

- to trigger adaptation directly on the flow itself. For example, the content of a flow may be compressed, transcoded, encrypted, classified, assigned a particular QoS treatment, marked, or rerouted in response to link constraints, network state, or application requirements [5]. 
- to trigger immediate or future network-wide management actions that are not necessarily targeted to exclusively affect the context-carrying flow. For example, a new flow may trigger the activation of a reserve link in order to guarantee sufficient bandwidth for future flows.

- to trigger network services that are specific or personalized for a user or group of users. A context tag may carry information about the user generating the flow, such as her profile or preferences. The network's response may be to offer these specific services to the user, or to automatically provide them in a transparent way. These new services may or may not directly affect the current flow.

- as a means to collect information for long-term purposes, without triggering any immediate adaptation or service, such as for traffic engineering or network optimization, or for consumption by cognitive or knowledge-based entities within the network [6].

Our approach involves tagging network flows with context obtained from sensors located mainly at end-hosts, by injecting packets that carry the flow's context within the flow itself. These context tags are preferably located at the start or within the first few packets of a flow, although there may be times where they may be reinserted anytime during the flow lifetime, especially to signal changes in the flow's context.

\section{Context-Tagging Architecture}

A diagram illustrating the main functional components in our scheme is show in Figure 1. Some of the components are based on a cognitive framework previously described in [7].

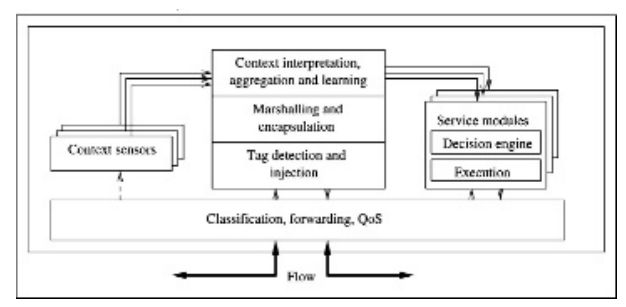

Fig. 1. Context tagging framework

\subsection{Tag Creation and Injection}

Sensors collect low-level context information, usually measurable data or lowlevel events. These are transformed into useful form through the process of interpretation, which may involve the application of an algorithm, comparison with a model, or the application of reasoning or other cognitive processes. Aggregation involves the examination of separate pieces of context information to generate new contextual knowledge. 
The context information is then passed down to the marshalling and encapsulation stage, where it is transformed into an Extensible Markup Language (XML) document [8]. The use of XML provides a rich and extensible means of representing context information, and allows the formal specification of languages governing the interpretation of such context. It is further encapsulated in a User Datagram Protocol (UDP) transport-layer datagram [9]. The use of UDP allows other context- "interested" hosts, including the end-destination of the flow, to detect or demultiplex out the context tag packet from the rest of the flow.

Further down, the tag injection and detection stage encapsulates the UDP datagram in an IP packet whose header contains the IP Router Alert Option as described in RFC 2113 for IPv4 [10], and RFC 2711 for IPv6 [11]. This option has the semantic "routers should examine this packet more closely."

Routers within the network may also inject context tags to describe information aggregated from multiple tags within a single flow, or to describe flow aggregates called macroflows. In some cases, such as within the Internet core, routers may be more interested in context descriptions of large macroflows rather than the individual constituent flows, for reasons of scalability. It may be also necessary for routers to inject context tags to describe any adaptation they may have performed on a flow or macroflows.

\subsection{Service Triggering}

Routers within the network detect the context tag by virtue of the Router Alert option in the IP header. Routers that either do not support the option, or do not recognize the context payload simply forward the packet to the next hop. At end-hosts, the context tag may be demultiplexed out of the flow by virtue of the UDP port number. If no equivalent context-processing process exists at the destination host the tag is either silently dropped, or an error message may be returned.

Once received, the context payload is extracted and sent up the stack, to the context interpretation and aggregation module. The context may either be used for long-term information gathering or learning, or may result in a specific adaptation being triggered. Service (including adaptation) modules subscribe to certain context values or events within the context interpretation module. When these events occur or the values are presented to the service module, a decision engine determines if the service or adaptation is executed. In the case of routers, the adaptation may be applied directly to the flow carrying the tag, or to a group of flows, or a larger-scale adaptation or management function may be invoked. Service modules on different nodes may communicate with each other and may operate in a coordinated fashion; however, the details of this operation is beyond the scope of this paper.

The architecture also allows end-hosts to receive and process context tags, and to contain adaptation components. In this case, the adaptation could be on the incoming or outgoing network flow, or on a user application, or influence some operation of the receiving device. 


\section{Deployment Strategy}

Our context-tagging scheme can be incrementally deployed on networks by progressively adding components that either support flow context sensing and tag generation, or provide context-triggered services and adaptation, or both. Context sensing functionality may be added to end-hosts or incrementally on network nodes such as routers, or dedicated boxes may be inserted within the network in order to inspect flows and inject context tags. For nodes that will provide context-triggered services, the service modules and the core router functionalities (classification, forwarding, QoS) do not necessarily have to be closely coupled; the context-related and adaptation functions could reside on a separate device "bolted" onto a conventional router, and SNMP [12] may be used to effect service execution. While there are obvious advantages in terms of performance and a wider range of functionalities are possible in the closely-coupled case, the loosely-coupled example is given here to illustrate that the scheme may be incrementally deployed on the existing Internet infrastructure.

\section{$5 \quad$ Initial Results}

A simple flow-generating application using libnet [13], a toolkit that allows lowlevel packet construction, handling and injection was designed and used to simulate the operation of our system and perform initial validation on some of its components. Our application generated a flow containing exactly one context tag packet carrying the Router Alert Option in the IP header and a rudimentary XML-formatted context payload encapsulated in UDP. The rest of the flow consisted of UDP datagrams carrying dummy payloads. The context tag packet was positioned well within the flow, rather than among the first few packets, so that the adaptation could be well-observed. The flow was sent through a Linux-based router that in turn ran a process that detected the context-tagged packet.

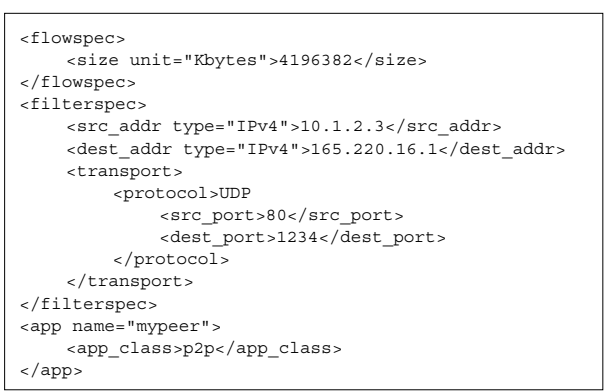

(a)

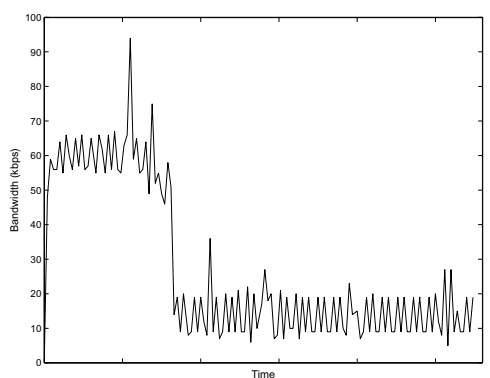

(b)

Fig. 2. (a) Context tag fragment. (b) Adaptation response on router 
On the Linux router, the tag was processed, triggering a simple adaptation. In this example, the specific adaptation was to map the flow into an appropriate traffic class. Within the context tag a component called the filterspec provided a function similar to a similarly-named component in the Resource Reservation Protocol (RSVP): a means to define the set of packets that constitute the flow described by the tag [14]. In this experiment our filterspec used a Linux u32 filter [15] descriptor that was directly parsed by the router and passed on to the adaptation module. In future implementations we expect to use a wider variety of mechanisms to specify filterspecs, including high-level descriptions that could be mapped by the context interpreter and service modules to specific classification mechanisms within the router. The adaptation module used the Linux iptables and tc mechanisms [15] to map the flow to a traffic class.

A fragment of the context tag is shown in Fig. 2a. The router's response to the context tag is shown in Fig. 2b. Prior to receiving the tag, the network accommodated the flow's full offered load (60 kbps average). Upon receiving the tag, the router effectively limited the bandwidth allocated to the flow to an average of $14 \mathrm{kbps}$.

\section{Related Work}

The network edge is a rich source of context information. End-hosts are in the best position to provide information on the applications that generate or consume traffic, the users whose activities drive these applications, and the computational and networking capabilities of the devices on which these applications run. Currently no widely deployed and sufficiently flexible mechanism exists for the edge to share context information within the network. Many context-aware systems employ publish-subscribe mechanisms that allow clients to obtain context information from sources $[3,16]$. Our approach however contemplates a more loosely-coupled, connectionless communications model between end-hosts and nodes.

While network- and transport-layer protocol headers contain rudimentary tags (fields) that provide some hints on the type of applications that generate the flow, or to some extent, the nature and characteristics of the flow's content, these tags are not expressive enough to encapsulate the rich context information available at the edge. Schemes that provide flow descriptions often focus exclusively on their QoS characteristics, rather than a more general description of flow context. Resource reservation protocols such as RSVP [14] and the experimental ST-II [17] use a flowspec to describe the characteristics of a flow, usually in terms of bandwidth, delay, and other QoS-related characteristics [18]. Other schemes such as the Session Description Protocol (SDP) [19] and Multipurpose Internet Mail Extensions (MIME) [20] deliver flow or session context to end-hosts rather than network nodes, and are limited to very specific application domains. 


\section{Conclusion and Future Work}

A key component needed to enable the deployment of context-aware networks is a method for sharing network context information. We have discussed a simple yet extensible and semantically-rich mechanism that tags flows through the network with context, delivering context directly to nodes along the unicast path or multicast tree. This allows network services, particularly adaptation services, to be triggered based on flow context. Our on-going work is focused on further validating our architecture, evaluating its performance, and exploring other application scenarios.

We are likewise currently exploring a parallel implementation of our components on an active networking [21] platform. We believe that such an approach will allow us to achieve a greater amount of flexibility through an on-demand deployment of context sensing, tagging, interpretation and adaptation components within the network.

Routers and end-hosts would be able to efficiently share context only if they subscribe to a common information model. An important component of our ongoing work is the development of an ontology and appropriate context-sharing languages.

Finally, as with any system that senses and distributes context information, there are concerns on security and privacy that we also hope to address in the near future.

Acknowledgment. This paper describes work partially undertaken in the context of the E-NEXT - Information Society Technologies (IST) FP6-506869 project. The IST program is partially funded by the Commission of the European Union. The views and conclusions contained herein are those of the authors and should not be interpreted as necessarily representing the E-NEXT project. Roel Ocampo acknowledges support by the Doctoral Studies Fellowship program of the University of the Philippines.

\section{References}

1. I. Foster. What is the Grid? A Three Point Checklist. Grid Today, July 2002.

2. J. Saltzer, D. Reed, and D. Clark. End-to-End Arguments in System Design. ACM Transactions on Computer Systems, November 1984.

3. A. K. Dey, D. Salber, and G. D. Abowd. A Conceptual Framework and a Toolkit for Supporting the Rapid Prototyping of Context-Aware Applications. HumanComputer Interaction (HCI) Journal, 16 (2-4), 2001

4. R. Braden, D. Clark, and S. Shenker. Integrated Services Architecture in the Internet: an Overview. Request for Comments 1633, June 1944.

5. M. Yarvis, P. Reiher and G. Popek. Conductor: A Framework for Distributed Adaptation. Proc. 7th Workshop on Hot Topics in Operating Systems, March 1999.

6. D. Clark, C. Partridge, J. Ramming, and J. Wroclawski. A Knowledge Plane for the Internet. Proc. ACM SIGCOMM 2003 Conference on Applications, Technologies, Architectures and Protocols for Computer Communications, August 2003. 
7. R. Ocampo and H. De Meer. Smart Wireless Access Points for Pervasive Computing. Proc. First Working Conference on Wireless On Demand Network Systems (WONS '04), Lecture Notes in Computer Science LNCS 2928, January 2004.

8. T. Bray, J. Paoli, C.M. Sperberg-McQueen, E. Maler, and F. Yergeau (editors). Extensible Markup Language 1.0 (Third Edition). W3C Recommendation 04 February 2004, http://www.w3.org/TR/REC-xml

9. J. Postel. User Datagram Protocol. Request for Comments 768, August 1980.

10. D. Katz. IP Router Alert Option. Request for Comments 2113, February 1997.

11. C. Partridge and A. Jackson. IPv6 Router Alert Option. Request for Comments 2711 , October 1999.

12. J. Case, M. Fedor, M. Schoffstall, J. Davin. A Simple Network Management Protocol (SNMP). Request for Comments 1157, May 1990.

13. M. Schiffman. Libnet. http://www.packetfactory.net/ Projects/Libnet/

14. L. Zhang, S. Deering, D. Estrin, S. Shenker, and D. Zappala. RSVP: A New Resource ReSerVation Protocol. IEEE Network, September 1993.

15. B. Hubert, T. Graf, G. Maxwell, R. van Mook, M. van Oosterhout, P. Schroeder, J. Spaans, P. Larroy (editors). Linux Advanced Routing and Traffic Control. http://www.lartc.org.

16. T. Kanter. A Service Architecture, Test Bed and Application for Extensible and Adaptive Mobile Communication. Proc. Personal Computing and Communication Workshop 2001 (PCC'2001), April 2001.

17. C. Topolcic. Internet Stream Protocol Version 2 (ST-II). Request for Comments 1190, October 1990.

18. C. Partridge. A Proposed Flow Specification. Request for Comments 1363, September 1992.

19. M. Handley, V. Jacobson. SDP: Session Description Protocol. Request for Comments 2327, April 1998.

20. N. Freed and N. Borenstein. Multipurpose Internet Mail Extensions Part Two: Media Types. Request for Comments 2046, November 1996.

21. D. L. Tennenhouse and D. J. Wetherall. Towards an Active Network Architecture. Computer Communication Review, 26(2), April 1996. 